\title{
Research of the Channel Estimation in Wireless Communication Systems
}

\author{
Lili Zhao ${ }^{a}$, Peng Zhang, Qicai Dong, Xiangyang Huang, Jianhua Zhao and Zeyu Su \\ No.12 Fan Gongting south street, Shandong, China
}

\begin{abstract}
Wireless communication technology has been developed rapidly after entering the 21 st century. Data transfer rate increased significantly as well as the bandwidth became wider and wider from $2 \mathrm{G}$ to $4 \mathrm{G}$ in wireless communication systems. Channel estimation is an import part of any communication systems; its accuracy determines the quality of the whole communication. Channel estimation methods of typical wireless communication systems such as UWB, $2 \mathrm{G}$ and $3 \mathrm{G}$ have been researched.
\end{abstract}

\section{Introduction}

According to the Federal Communications Commission, a signal with a relative bandwidth of more than $20 \%$ at $-10 \mathrm{~dB}$ or an absolute bandwidth exceeding $500 \mathrm{MHz}$ is called ultra-wideband. It is defined as follows:

Relative bandwidth:

$$
B=\frac{\left(f_{h}-f_{l}\right)}{\frac{\left(f_{h}+f_{l}\right)}{2}} \times 100 \%=\frac{2\left(f_{f_{h}}-f_{l}\right)}{\left(f_{h}+f_{l}\right)} \times 100 \%>20 \%
$$

Or absolute bandwidth: $B=\left(f_{h}-f_{l}\right) \geq 500 M H Z$.

In the formula, $f_{h}, f_{l}$ is the maximum limiting frequency and the minimum frequency corresponding to the power spectral density of the signal at $-10 \mathrm{~dB}$. $(\mathrm{fh}+\mathrm{fl}) / 2$ is the central frequency. In ultra-wideband applications, FCC prescribes that the band of $3.1 \mathrm{GHZ}$ 10.6GHZ allows access to the civilian sector. FCC also specifies UWB radiation power to avoid interference with other systems.

Ultra-wideband (UWB) systems are divided into two types: UWB based on pulse form using direct sequence spread spectrum technology, that is DS-UWB; and UWB based on traditional OFDM technology, namely MBOFDM-UWB. MB-OFDM-UWB system is studied in this paper.

The ultra-wideband (UWB) system uses extremely short narrow-band pulses to transmit data, has high bandwidth in the frequency domain, and enables highspeed data transmission for short-range wireless communication. UWB system also has other characteristics, such as low power consumption, low power spectrum density and good ability to resist multipath interference. UWB system is widely used in many fields. It is suitable for indoor wireless communication, ranging, short-range accurate positioning and so on

UWB systems can achieve very high transmission rates (above 100Mbps). When UWB operates in high-rate mode, its transmission distance is relatively close, generally between 10-20 meters; when operating in lower-rate mode, the transmission distance will become longer. The UWB system also has the function of locating, and the UWB in the form of pulse has strong perspective ability, and can communicate or locate through the thick walls of several layers.

\section{Introduction to Compressed Sensing Technology}

In a communication system, if the receiving end wants to recover the signal of the transmitting end without distortion, the receiving end must satisfy the Nyquist sampling theorem when sampling, that is, the sampling rate of the analog signal cannot be less than twice the signal bandwidth. In the past half century, the Nyquist sampling theorem has been used for the acquisition, processing, transmission, and storage of all images and signals.

The frequency of ultra bandwidth system is above $\mathrm{GHz}$, according to the sampling theorem, the receiver is required to have a very high sampling rate, which will produce a huge amount of data. In order to reduce the cost of transmission or storage, only a small amount of important data can be retained. This results in a serious waste of sampling resources.

Donoho, Candes and Taoundefineds new theory of Compressed Sensing (CS) can solve this problem well. The core idea of the theory of compressed sensing: according to the sparsity of the signal, the highdimensional signal is projected into the low-dimensional space by using a specific matrix, and the original signal is restored by a linear or nonlinear reconstruction algorithm. Simply put, if the signal is sparse (or sparse through a certain transform), then the sparse signal can be reduced

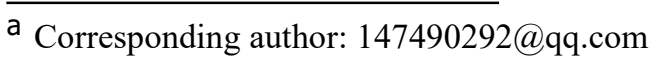


by an uncorrelated measurement matrix, and then the original signal is recovered by the reduced dimension data. Compressed sensing theory consists of three parts:

(1) Sparse representation of the signal: The so-called "sparse" means that the signal itself has few non-zero elements or a small value that is mostly approximately zero after the transformation, with only a small number of elements being larger.

(2) The design of the observation matrix: Find a matrix that is uncorrelated with orthogonal bases, which is capable of converting a high-dimensional signal to a low-dimensional representation, that is, a process that obtains fewer observations.

(3) Signal reconstruction algorithm: Designing highquality reconstruction algorithms to accurately recover the original signal from fewer observations is also a key technology in compressed sensing.

\section{Ultra-wideband channel estimation method}

Ultra-wideband systems are limited by FCC power, resulting in a very short transmission distance (only 0 to $10 \mathrm{~m}$ ). Most of the literature studies the ultra-wideband indoor channel model. The UWB channel model is divided into a path loss model and a multipath channel model. This paper uses a multipath channel model.

Among the many channel models, the models that can well represent the characteristics of the ultra-wideband channel are $\Delta-K$ model, $S-V$ model and IEEE802.15.3a channel model. Among them, IEEE802.15.3a is improved on the basis of $S-V$. This channel model fits the measured data well, and it is closer to the measured value in terms of RMS extended delay and average extended delay.

The UWB channel model studied in this paper is released by the IEEE802.15.3a working group. There are four UWB channel types of CM1-CM4. The description of each channel is shown in Table 1. The research and simulation in this paper is carried out under the CM1 channel. Figure 1 shows the impulse response of the CM1 channel model.

Table 1. UWB channel parameter statistics table

\begin{tabular}{|c|c|c|c|c|}
\hline Channel model & CM 1 & CM 2 & CM 3 & CM 4 \\
\hline NSEC & 5.05 & 10.38 & 14.18 & \\
\hline NSEC & 5.28 & 8.03 & 14.28 & 20 \\
\hline$\Lambda(1 / \mathrm{nsec})$ & 0.0233 & 0.4 & 0.0667 & 0.0667 \\
\hline$\lambda(1 / \mathrm{nsec})$ & 3.75 & 1 & 3 & 3 \\
\hline$\Gamma$ & 7.1 & 5.2 & 14.93 & 17 \\
\hline$\gamma$ & 4.37 & 6.5067 & 7.03 & 12 \\
\hline$\sigma(\mathrm{dB})$ & 4.8 & 4.8 & 4.8 & 4.8 \\
\hline Environment & LOS $(0-4 \mathrm{~m})$ & NLOS $(0-4 \mathrm{~m})$ & NLOS $(4-10 \mathrm{~m})$ & Extreme NLOS \\
\hline
\end{tabular}

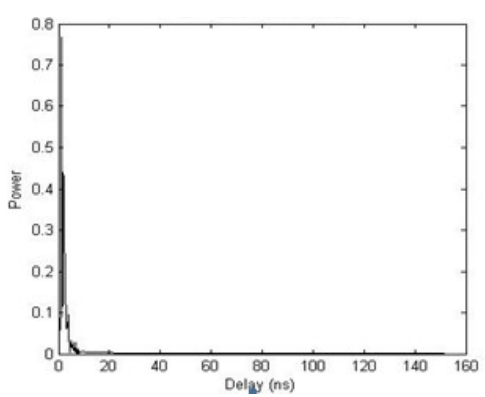

Figure 1. Channel impulse response of the CM1 channel model

\subsection{Traditional UWB channel estimation method}

The UWB system utilizes a training sequence known at the receiving end, according to the following formula:

$$
Y=X H+Z
$$

Figure 2 shows the baseband system model of UWB on the MATLAB platform.

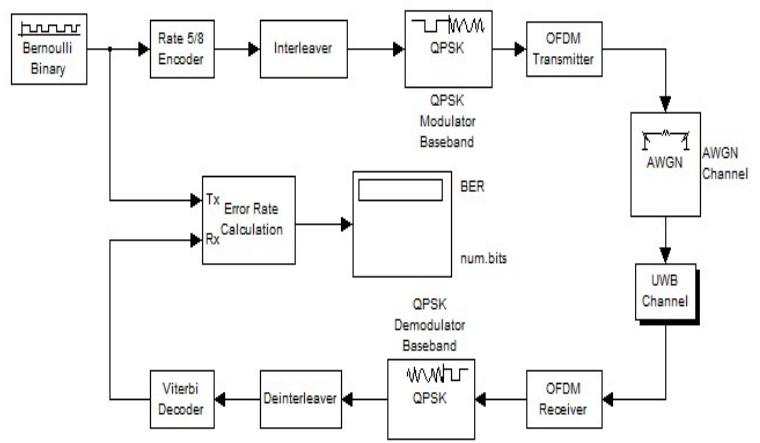

Figure 2. MATLAB platform UWB system diagram

The transmitter of the UWB system inserts six training sequences into each symbol of the OFDM for channel estimation. In frequency domain, after estimating the position of the training sequence, the average value is taken as the frequency domain response of a frame of OFDM symbols.

\subsection{Application of Compressed Sensing in UWB}

This method uses compressed sensing in time domain to receive the information received by the receiver:

$$
r=x * h+n=A h+n
$$

Where $\mathrm{h}$ is the channel time domain impulse response and $\mathrm{n}$ is the noise.

$$
\mathrm{A}=\left[\begin{array}{ccc}
x_{0} & \mathrm{~L} & 0 \\
x_{1} & \mathrm{O} & \mathrm{M} \\
\mathrm{M} & \mathrm{O} & x_{0} \\
x_{N-1} & \mathrm{O} & x_{1}
\end{array}\right]
$$

If there is an M-dimensional observation matrix, $\mathrm{A}$ is reduced from $\mathrm{N}$ to $\mathrm{M}$, where $\mathrm{M}<<\mathrm{N}$ :

$$
y=\Phi A h=\Theta h
$$

If $\Theta=\Phi A$ satisfies the constrained equidistant side (RIP) and $\mathrm{h}$ is sparse, then compression sensing can be used to reconstruct $h$.

The matrix $\mathrm{A}$ is a Topplitz matrix, and a new matrix consisting of a number of rows spaced from the tops of the Topplitz matrix is called a quasi-topplez matrix. The form is as follows, the Topplitz matrix on the left and the quasi-topplez matrix on the right. 


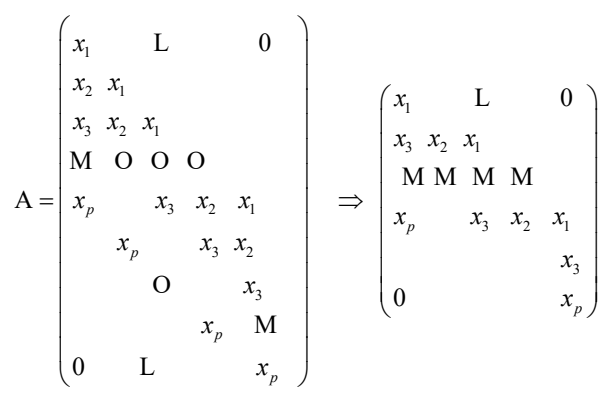

Reference [6] pointed out that if the elements in the topology are obeying a normal distribution with a mean of 0 , then the quasi-top Pritz matrix constructed by this topology can also be used for compressed sensing.

The training sequence needs to follow a normal distribution of zero mean. Figure 4 shows the channel estimation and equalization portion of the receiver.

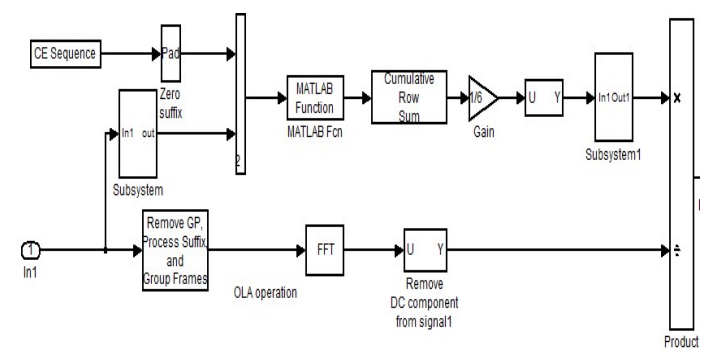

Figure 4. UWB channel estimation equalization part

The CE Sequence module in Figure 4 is a training sequence added by the sender in the time domain. The following module extracts the training sequence of the receiver. The Matlab Function module is an OMP reconstruction algorithm in which channel estimation is performed. The latter module averages the channel estimates and then equalizes the received signals. Figure 5 shows the curve of BER changing with signal-to-noise ratio (SNR) obtained by simulation. It can be seen from the simulation diagram that the BER of the system decreases gradually with the increase of signal-to-noise ratio $(\mathrm{SNR})$. When the BER of $\mathrm{SNR}=6$ reaches $10 \sim(-6)$, it shows that this method can estimate the impulse response of the channel well.

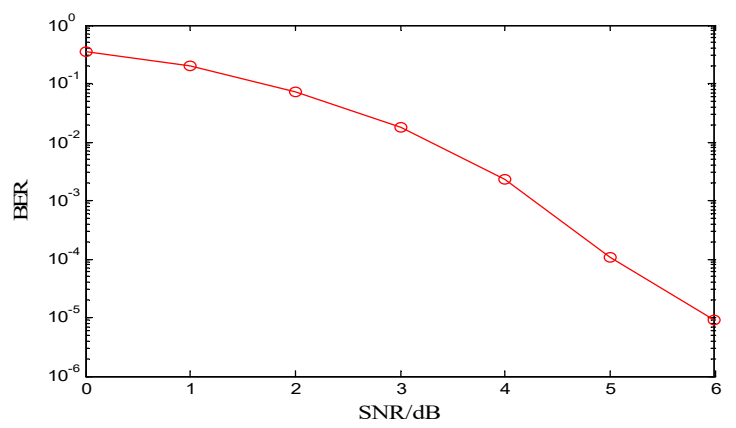

Figure 5. Bit Error Rate Graph

Figure 6 is a contrast diagram of the reconstructed channel and the original channel at $\mathrm{snr}=6$. As can be seen from this graph, the reconstructed channel is very close to the original channel, which also shows that the method can estimate the impact response of the channel Well.

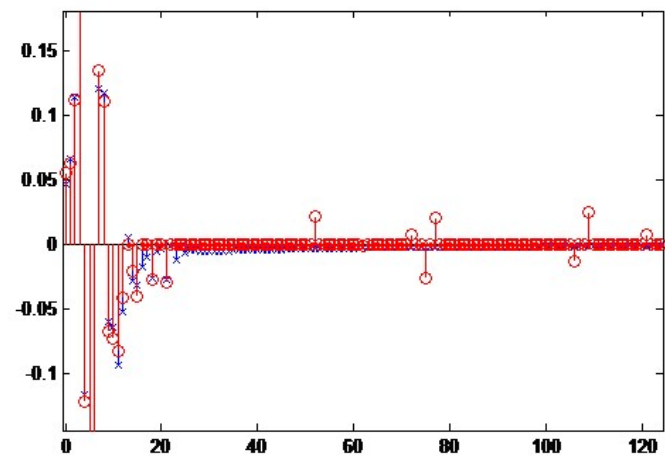

Figure 6. Comparison of reconstructed channel and original channel

It can be seen from the simulation diagram that the method of compressive sensing in the time domain can well estimate the frequency domain response of the channel.

\section{Conclusion}

This paper introduces the application of the theory of compression perception in Ultra-wideband channel estimation, is a time domain using known training sequence, Time-domain Channel estimation method in the time domain, need to add to the transmitter to meet the known conditions of the training sequence. Because the time domain method needs to join the known training sequence, it needs to change the system structure, and the system has been stipulated in the standard, the disadvantage of this method is to add a training sequence which conforms to the known condition in the time domain, and the implementation is more Complicated. In the future study, we try to use 6 training sequence symbols in the frequency domain for channel Estimation.

\section{References}

1. Yang L, Giannakis G B. Ultra-wideband communications: an idea whose time has come [J]. Signal Processing Magazine, IEEE, 2004, 21(6): 2654.

2. Donoho D L. Compressed sensing [J]. Information Theory, IEEE Transactions on, 2006, 52(4): 12891306.

3. Bajwa W U, Haupt J, Sayeed A M, et al. Compressed channel sensing: A new approach to estimating sparse multipath channels [J]. Proceedings of the IEEE, 2010, 98(6): 1058-1076.

4. Tropp J A, Gilbert A C. Signal recovery from random measurements via orthogonal matching pursuit $[\mathrm{J}]$. Information Theory, IEEE Transactions on, 2007, 53(12): 4655-4666.

5. Molisch A F, Balakrishnan $\mathrm{K}$, Chong $\mathrm{C} \mathrm{C}$, et al. IEEE 802.15. 4a channel model-final report [J]. IEEE P802, 2004, 15(04): 0662.

6. Li Shutao, Weidan. A review of compression sensing [J]. Journal of automation, 2009, 35 (11): 1369-1377. 